\title{
Effect of Conditioning on Adhesion of Glass lonomer Cements to Dentin
}

\author{
${ }^{1}$ Aparna Ramachandran, ${ }^{2} \mathrm{~B}$ Jyothi Lekshmi, ${ }^{3}$ Rajesh Pillai, ${ }^{4}$ Nettiyat O Varghese, ${ }^{5}$ Afzal A Salim, ${ }^{6}$ U-Nu Sujathan
}

\section{ABSTRACT}

Aim: To compare the shear bond strength (SBS) to dentin, of three different glass ionomer cements (GIC): Ketac Universal (with and without conditioner), Fuji IX, and Ketac Molar.

Materials and methods: Forty-eight extracted human maxillary premolars were selected. Depth holes measuring $1.5 \mathrm{~mm}$ were drilled in the deepest part of central fossa of each tooth sample. Occlusal surfaces were ground on a trimmer to expose flat dentinal surface and polished. The specimens were then embedded in autopolymerizing acrylic resin. Groups I, III, and IV specimens were restored with Ketac Universal, Fuji IX, and Ketac Molar respectively after conditioning. Group II specimens were restored with Ketac Universal without conditioning. A cellophane tube of diameter $4 \mathrm{~mm}$ was used to prepare GIC restorations. The samples were kept in water bath at $37^{\circ} \mathrm{C}$ for 24 hours. Each GIC specimen was loaded in a universal testing machine (INSTRON) with a crosshead speed of $0.5 \mathrm{~mm} / \mathrm{min}$. The bond strength (MPa) was calculated by dividing the shear force in Newton $(\mathrm{N})$ by area of adhesion $\left(\mathrm{m}^{2}\right)$.

Statistical analysis: The results were statistically analyzed by Welch test and analysis of variance (ANOVA) test with pairwise "t" test.

Results: The mean value of SBS was found to be highest for Ketac Molar, followed by Ketac Universal with conditioner, and Fuji IX GIC, and was lowest for Ketac Universal without conditioner.

Conclusion: Ketac Universal, when used without conditioner, showed significant values of SBS, but is less than other conventional GICs.

Keywords: Fuji IX, Ketac Molar, Ketac Universal, Shear bond strength.

How to cite this article: Ramachandran A, Lekshmi BJ, Pillai R, Varghese NO, Salim AA, Sujathan U-Nu. Effect of Conditioning on Adhesion of Glass lonomer Cements to Dentin. Cons Dent Endod J 2018;3(1):6-10.

Source of support: Nil

\section{Conflict of interest: None}

\footnotetext{
${ }^{1,2}$ Postgraduate Student, ${ }^{3}$ Professor and Head, ${ }^{4}$ Prinicipal ${ }^{5}$ Professor, ${ }^{6}$ Reader

${ }^{1-6}$ Department of Conservative Dentistry and Endodontics, PMS College of Dental Sciences \& Research, Thiruvananthapuram Kerala, India

Corresponding Author: Aparna Ramachandran, Postgraduate Student, Department of Conservative Dentistry and Endodontics, PMS College of Dental Sciences \& Research Thiruvananthapuram, Kerala, India, Phone: +918921333398 e-mail: aparnanambiar22@gmail.com
}

\section{INTRODUCTION}

The adhesiveness of restorative materials plays a key role in the success of current restorative technique. It helps in eliminating secondary caries, marginal discolorations, microleakage, and pulpal damage. Adhesion of a material can be assessed by the determination of tensile and SBS. ${ }^{1,2}$ Bonding not only improves retention but also stabilizes a restoration without excessive removal of sound tooth structure. The need for providing large undercuts and auxiliary retentive aids is eliminated. Adhesive aids in transmission and distribution of functional stresses across the bonded interface which helps in reinforcing weakened tooth tissue. There is less chance for marginal gaps to occur due to polymerization stress when there is a strong adhesion between tooth and restorative materials. $^{3}$

Dentin adhesion is more difficult and unpredictable because of complex histologic structure and variable composition. ${ }^{4}$ In contrast to regular arrangement of hydroxyl apatite crystals, dentinal hydroxyl apatite is randomly arranged in an organic matrix that consists primarily of collagen. ${ }^{5}$

Debris that form while the tooth is cut can get burnished on to the surface, resulting in smear layer formation which can affect dentin bonding. Its thickness and appearance vary with the specific substrate and the type of cutting instrument used. 6,7

Self-adhesiveness without any need for surface pretreatment is an important property of GIC. Bonding can be significantly improved if the surface is pretreated with a weak polyalkenoic acid conditioner. ${ }^{8,9}$

A significant hike in the adhesion property of Alumino silicate polyacrylic acid glass ionomer was noted by Powis et $\mathrm{al}^{10}$ after treating with polyacrylic, tannic acid, and dodicin solution for 30 seconds, except $\mathrm{H}_{2} \mathrm{O}_{2}$.

The basic phenomenon behind the bonding is the ionic attraction between two carboxyl ( $\left.\mathrm{COO}^{-}\right)$groups in the cement to the calcium $\left(\mathrm{Ca}^{++}\right)$present in enamel and dentin. ${ }^{11}$ Traditional GICs were water-based materials which was set by an acid-base reaction between a polyalkenoic acid and a fluoro-alumino-silicate glass.

High powder-liquid ratio conventional GICs were marketed in the mid- to late 1990s. It was alternatively termed "packable" or "high-viscosity" GICs. These 


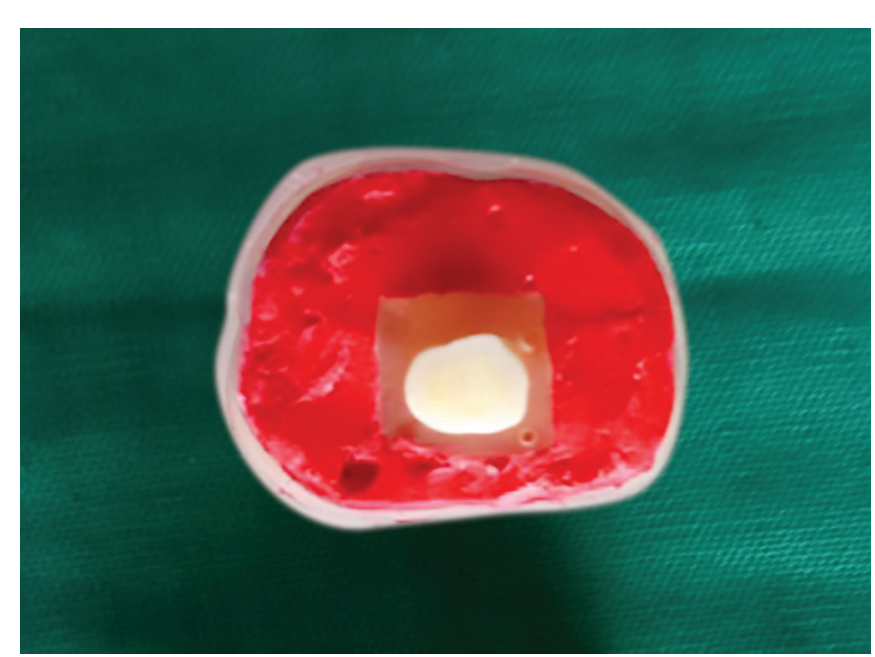

Fig. 1: Mounting specimens

products (e.g., Ketac Molar, 3M/ESPE, Seefeld, Bavaria, Germany; Chemflex, Dentsply, York, Pennsylvania, USA; Fuji IX and Fuji IX GP, GC International) were well known for their use in small cavities in deciduous teeth, temporary restorations, liner/base applications, and in the "Atraumatic Restorative Treatment" technique. ${ }^{11} \mathrm{Ketac}^{\mathrm{TM}}$ Universal Glass Ionomer Restorative is an innovative initiative by 3M ESPE Dental. The manufacturer claims that the material does not require preconditioning of the cavity for sufficient bonding. Thus, steps in restoration placement are reduced but compressive strength and surface hardness are not compromised, and reported to be more than several clinically proven glass ionomers that require a coating. The current study aims at comparing the adhesiveness of newly marketed Ketac Universal with Ketac Molar and Fuji IX.

\section{MATERIALS AND METHODS}

Forty-eight extracted permanent human maxillary first premolar teeth were chosen, cleaned with ultrasonic scaler, and stored in distilled water at room temperature, the preferred method of storage with least negative influence on the measured bond strength of resin composite to dentin. Depth holes measuring $1.5 \mathrm{~mm}$ were drilled in the deepest part of central fossa of each tooth with the help of a round diamond bur to standardize the depth of dentin. All the forty-eight samples were then ground on an orthodontic trimmer to expose a flat dentinal surface which was followed by manual polishing of the dentinal surface with wet 600 grit silicone carbide paper. The specimens were embedded in autopolymerizing acrylic resin in the custom-made alginate molds (Fig. 1).

The specimens were placed perpendicular to the acrylic resin surface. Care was taken to keep the experimental surfaces free of resin (Fig. 2).

To prepare the specimens for SBS measurement, a cylindrical split Teflon mold ( $3 \mathrm{~mm}$ in diameter, $3 \mathrm{~mm}$ in height) was used and each material was loaded into the mold.

Specimens were divided into four groups.

Group I : Ketac Universal without conditioner

Group II: Ketac Universal with conditioner

Group III: Ketac Molar with conditioner

Group IV: FUJI IX with conditioner

For conditioning of dried dentinal surface, $10 \%$ polyacrylic acid was used for 10 seconds. Powder and liquid were mixed at a ratio of 3:1. After 30 seconds of placement of GIC, nylon cylinders were removed. This was followed by application of GC Fuji varnish on the entire surface. The method was the same for all the three groups except group I, which required no conditioning and GIC was directly applied onto the dentin surface.

\section{Calculation of SBS}

Each specimen was loaded in a universal testing machine (INSTRON) with a crosshead speed of $0.5 \mathrm{~mm} / \mathrm{min}$ (Fig. 3). The bond strength (in $\mathrm{MPa}$ ) was calculated by dividing the shear force in Newton $(\mathrm{N})$ by the area of adhesion.

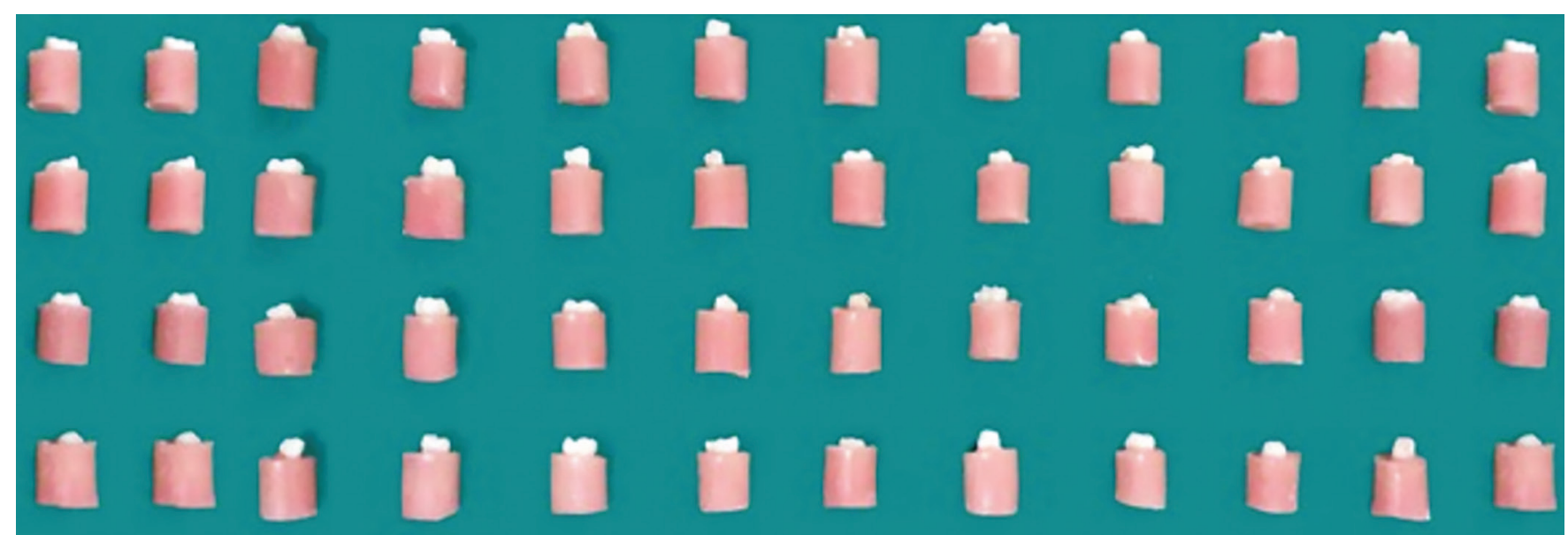

Fig. 2: Specimens embedded in acrylic 


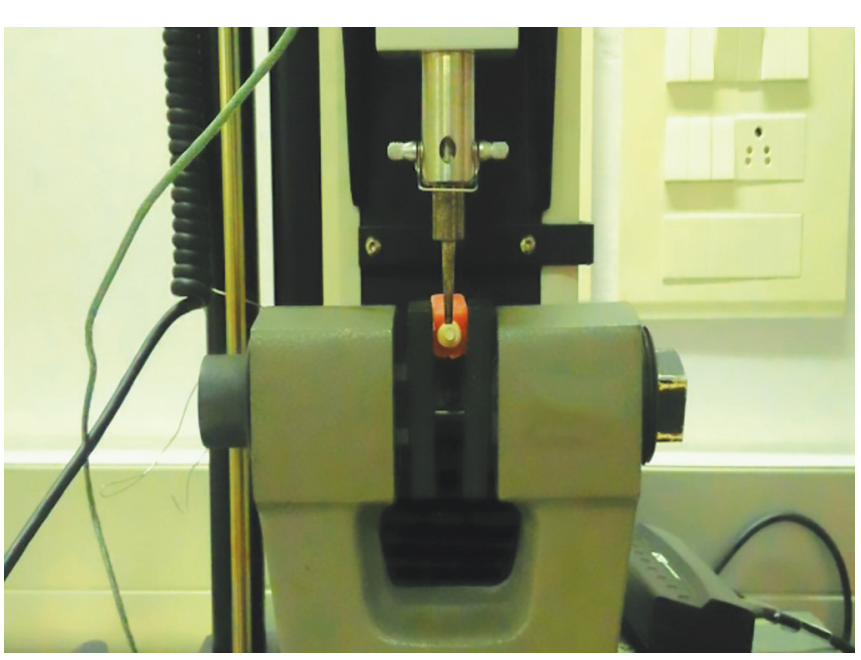

Fig. 3: Specimen loaded in universal testing machine

Table 1: Mean value of SBS of test specimens

\begin{tabular}{ll}
\hline GIC type & Mean SBS (MPa) \\
\hline Ketac Universal with conditioner (KUC) & 3.3 \\
Ketac Universal without conditioner (KU) & 3.02 \\
Ketac Molar (KM) & 3.4 \\
FUJI IX (FIX) & 3.2 \\
\hline
\end{tabular}

In this study, the shear force was applied perpendicular to the tooth surface.

\section{Statistical Analysis}

The data collected were tabulated and was subjected to statistical analysis. Mean and standard deviations were calculated for each group and analyzed using a one-way ANOVA. Student's " $\mathrm{t}$ " test was used to identify differences between the two groups.

\section{RESULTS}

Shear bond strength (in MPa) was calculated according to the following formula:

$$
\text { Stress }=\frac{\text { Failure load }(\mathrm{N})}{\text { surface area }\left(\mathrm{mm}^{2}\right)}
$$

Test result showed that the mean value of SBS was highest in group III (Table 1) and test results were plotted graphically (Graph 1).

\section{DISCUSSION}

Ability of restorative material to withstand the dislodging forces within oral cavity depends upon the adhesiveness of material to dentin surface which is an important deciding factor in the clinical success of restorative material. ${ }^{3}$ Even though compressive and tensile strengths are important parameters since the major dislodging forces at the tooth restoration interface have shearing effect, we have evaluated SBS in the present study. Higher SBS implies better bonding of the material to tooth.

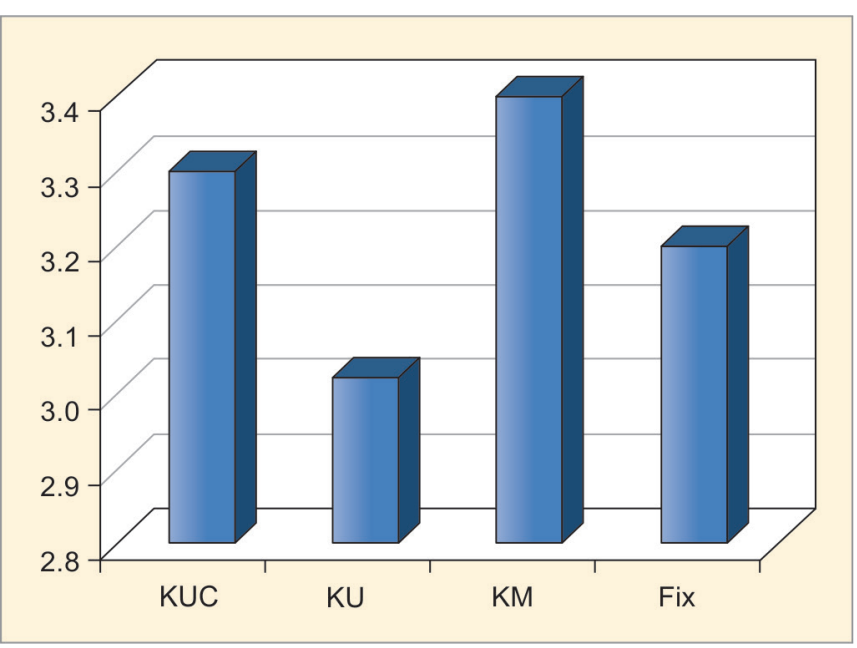

Graph 1: Mean value of SBS

Studies testing SBS of GICs to dentin have found values ranging from 1.32 to $4.10 \mathrm{MPa}^{12}$ In the present study, the mean value of SBS was found to be highest for Ketac Molar (3.4 MPa), followed by Ketac Universal with conditioner (3.3 MPa) and type IX GIC (3.2 MPa), and was lowest for Ketac Universal without conditioner (3.02 $\mathrm{MPa}$ ). All the intercomparisons between various groups were also found to be highly significant. The results are in accordance with the study done by Carvalho et $\mathrm{al}^{12}$ regarding SBS of three GIC to enamel and dentin where they found that GIC that had the highest mean bond strength to both enamel and dentin was Ketac Molar Easymix, followed by Fuji IX and Maxxion.

Ketac Universal restorative is a bulk filling material having inherent radiopacity. Making it unique is its advantage that there is no need for additional coating or conditioning for adhesion due to the presence of sufficient free acid in cement to dissolve the smear layer at the time of placement, making it an ideal option for treating pediatric and geriatric patients. ${ }^{13}$

Fuji IX GIC is known as high viscous, condensable, or packable GIC. Higher strength, greater wear resistance, and flexural strength as compared with conventional GICs are attained because of smaller glass particle size and higher powder-liquid ratio. Fuji IX GIC is less moisture-sensitive and more resistant to dissolution than conventional GIC.

Ketac $^{\mathrm{TM}}$ Molar is a packable, high-viscosity, condensable, wear-resistant glass ionomer solution. In order to improve the mechanical properties and making it more packable, manufacturers have increased the viscosity by reducing the filler size.

The increase in bonding efficiency of GIC after conditioning must be partially attributed to

- Cleaning effect by which loose cutting debris are removed. 
- Increase in surface area due to the partial demineralization effect whereby microporosites for micromechanical interlocking or hybridization are exposed.

- Chemical interaction of polyalkenoic acid with residual hydroxyapatite. ${ }^{8}$

Polyacrylic acid and phosphoric acid at different concentrations have been evaluated as a pretreatment option to GICs and resin-modified GICs in order to adhere it efficiently to the dentin surface. ${ }^{14}$ Powis et al ${ }^{10}$ believed that it increases wettability of dentin surface and improves ion exchange with the cement, thus suggesting its use as dentin conditioner prior to placement of chemically set GIC. In the absence of any pretreatment, the intermediate layer incorporated only smear remnants and no demineralization was observed in the underlying intact dentin. ${ }^{15}$

Contradictory studies by Peutzfeldt and Asmussen ${ }^{16}$ reported that polyacrylic acid pretreatment improves the bond of GIC to rough dentin surfaces with thicker smear layers, but not for smoother dentin surfaces with thinner smear layer.

The underlying demineralized collagen may not be completely infiltrated by high molecular weight polyacrylic acid in chemically cured GICs. The situation may even be worsened by the collapse of the demineralized collagen network when acid-etched dentin is desiccated before GIC placement. Based on ultrastructural observations, there may be a deterioration of the GlC-dentin bond with the use of more aggressive pretreatment protocols that leave a bed of denuded collagen within the subsurface of demineralized dentin. ${ }^{15}$ More aggressive acid pretreatment protocol results in removal of the smear plugs and increases the permeability of dentin and outward flux of dentinal fluids during bonding. ${ }^{17}$

As per the current study results, newly marketed GIC Ketac Universal showed better results when used with conditioner, but showed significantly comparable result even in the absence of conditioner when compared with Ketac Molar and Fuji IX.

\section{CONCLUSION}

The following conclusions were drawn from present study:

- All the restorative materials used in the study showed significant values of SBS.

- The mean value of SBS was found to be highest for Ketac Molar, followed by Ketac Universal with conditioner, and type IX GIC, and was lowest for Ketac Universal without conditioner GIC.

\section{CLINICAL SIGNIFICANCE}

The property of adhesion serves as a useful tool in the retention of GICs within the tooth and also reduces or eliminates any chance of future marginal leakage. Thus, potentially harmful microorganisms are unable to enter the space under the restoration to promote dental decay. ${ }^{18}$

The use of acid conditioners for GIC pretreatment irrespective of whether they are rinsed or not before bonding invariably results in demineralization of intact dentin. There may be a deterioration of the GlC-dentin bond with the use of more aggressive pretreatment protocols that leave a bed of denuded collagen within the subsurface of demineralized dentin. In geriatric and pediatric patients, reducing the steps in placing restoration and thereby reducing the time consumed gives positive benefits.

\section{REFERENCES}

1. Murthy SS, Murthy GS. Comparative evaluation of shear bond strength of three commercially available glass ionomer cements in primary teeth. J Int Oral Health 2015 Aug;7(8): 103-107.

2. Knight GM, McIntyre JM, Mulyani. The effect of silver fluoride and potassium iodide on the bond strength of auto cure glass ionomer cement to dentine. Aust Dent J 2006 Mar;51(1):42-45.

3. Manuja N, Pandit IK, Srivastava N, Gugnani N, Nagpal R Comparative evaluation of shear bond strength of various esthetic restorative materials to dentin: an in vitro study. J Indian Soc Pedod Prev Dent 2011 Jan-Mar;29(1):7-13.

4. Swift EJ Jr, Perdigao J, Heymann HO. Bonding to enamel and dentin: a brief history and state of the art, 1995. Quintessence Int 1995 Feb;26(2):95-110.

5. Aasen SM. History of dentinal bonding. Esthet Dent Update 1990;1:43-46.

6. Eick JD, Wilko RA, Anderson CH, Sorensen SE. Scanning electron microscopy of cut tooth surfaces and identification of debris by use of electron microprobe. J Dent Res 1970 Jun;49(6):1359-1368.

7. Gwinnett AJ. Smear layer: morphological considerations. Oper Dent (Suppl) 1984;3:2-12.

8. Van Meerbeek B, De Munck J, Yoshida Y, Inoue S, Vargas M, Vijay P, Van Landuyt K, Lambrechts P, Vanherle G. Buonocore Memorial Lecture. Adhesion to enamel and dentin: current status and future challenges. Oper Dent 2003 MayJun;28(3):215-235.

9. Inoue S, Van Meerbeek B, Abe Y, Yoshida Y, Lambrechts P, Vanherle G, Sano H. Effect of remaining dentin thickness and the use of conditioner on micro-tensile bond strength of a glass-ionomer adhesive. Dent Mater 2001 Sep;17(5):445-455.

10. Powis DR, Folleras T, Merson SA, Wilson AD. Improved adhesion of a glass ionomer cement to dentin and enamel. J Dent Res 1982 Dec;61(12):1416-1422.

11. Tyas MJ, Burrow MF. Adhesive restorative materials: a review. Aust Dent J 2004 Sep;49(3):112-121.

12. Carvalho TS, van Amerongen WE, de Gee A, Bönecker M, Sampaio FC. Shear bond strengths of three glass ionomer cements to enamel and dentine. Med Oral Patol Oral Cir Bucal 2011 May;16(3):e406-e410.

13. $\operatorname{Ketac}^{\mathrm{TM}}$ Universal Glass Ionomer Restorative Technical Data Sheet. 
14. Poggio C, Beltrami R, Scribante A, Colombo M, Lombardini M. Effects of dentin surface treatments on shear bond strength of glass-ionomer cements. Ann Stomatol (Roma) 2014 Mar;5(1):15-22.

15. Tay FR, Smales RJ, Ngo H, Wei SH, Pashley DH. Effect of different conditioning protocols on adhesion of a GIC to dentin. J Adhesive Dent 2001 Summer; 3(2):153-167.
16. Peutzfeldt A, Asmussen E. Effect of polyacrylic acid treatment of dentin on adhesion of glass ionomer current. Acta Odontol Scand 1990 Oct;48(5):337-341.

17. Ciucchi B, Bouillaguet S, Holz J, Pashiey D. DentinaI fluid dynamics in human teeth, in vivo. J Endod 1995 Apr;21(4):191-194.

18. Sidhu SK, Nicholson JW. A review of glass-ionomer cements for clinical dentistry. J Funct Biomater 2016 Jun;7(3):E16. 